\title{
CONCORD: A UAV Conflict Resolution System using Correlated Equilibrium based Decision Making
}

\author{
Lima Agnel Tony* and Debasish Ghose ${ }^{\dagger}$ \\ Indian Institute of Science, Bangalore-560012, India \\ Animesh Chakravarthy \\ University of Texas at Arlington, Texas-76019, USA
}

\begin{abstract}
Unmanned Aerial Vehicles (UAVs) are in demand for a wide range of applications. A traffic management system similar to the Air Traffic Control (ATC) is necessary to handle these vehicles efficiently. The complexity associated with such a system will increase with the size of airspace and the number of vehicles using it at any given instant of time. The need for collision avoidance among UAVs is quite significant in this context. This paper presents the CONCORD, which is a UAV conflict resolution system that uses the notion of correlated equilibrium. Correlated equilibrium is a popular solution concept in game theory, which improves upon Nash equilibrium solution and gives better expected payoff to every player. In this work, an intelligent semi-autonomous conflict resolution subsystem is proposed which could be a part of UAV traffic management system that can handle multiple UAV conflicts effectively.
\end{abstract}

\section{Introduction}

Small size, minimum cost, and a variety of other factors, have led to actual and anticipated deployment of UAVs for various applications from defense systems [1] to unmanned air transport [2] to large scale disaster management [3]. As the number of vehicles increase, well defined rules are desired to take care of various aspects like vehicle safety, data privacy, national security preservation, segregation with manned vehicles, firewall strengthening, dedicated communication bandwidth, etc. Each aspect needs an in-depth analysis to ensure proper solution and error free implementation. The primary concern would be the safety of the UAVs and other vehicles which share the airspace. When it comes to manned aircraft, Air Traffic Control (ATC) takes care of vehicle routing using information regarding all vehicle movements within its authority. This is a system that requires human supervision for error free execution. As the density of UAVs increases in the airspace, it is conceivable that separate air ways or corridors will be identified for UAVs with separate landing and take-off airports. In which case, a similar mechanism as ATC needs to be implemented

\footnotetext{
* Research Scholar, Guidance, Control and Decision Systems Laboratory, Department of Aerospace Engineering

${ }^{\dagger}$ Professor, Guidance, Control and Decision Systems Laboratory, Department of Aerospace Engineering

$¥$ Associate Professor, Dept. of Mechanical \& Aerospace Engg., University of Texas at Arlington
} 
for UAVs which could be semi or fully automated. As reported in [1], a fully autonomous system addressing all aspects of UAV autonomy is a long term goal which is far into the future. An immediately realizable system would be a semi-autonomous safety mechanism which would receive inputs from a third party conflict resolution system to regulate the motion of the vehicles. This paper proposes such a system which acts as a third party mediator between the UAVs to resolve conflicts in an optimal manner using the concept of correlated equilibrium in game theory. The system is named CONCORD (A UAV Conflict Resolution System using Correlated Equilibrium based Decision Making).

One of the most common solution concepts in game theory is Nash equilibrium [4]. For such games, the equilibrium could be achieved by pure or mixed strategies, where strategy mixing is based on independent events and any unilateral deviation by any of the players would not result in an improved payoff. Aumann [5, 6] introduced the concept of correlated equilibrium which defined a more general solution method where the players mix their strategies in either independent or dependent fashion and obtain better payoffs. Mixed strategy Nash equilibrium is also a correlated equilibrium in the sense that the players choose actions based on independent events. It is proved in [5] that the set of correlated equilibrium contains the set of Nash equilibrium. Correlated equilibrium has been applied in various fields, especially in communication. Optimal spectrum sharing using correlated equilibrium is discussed in [7--9] while [10] consider a file sharing game based on correlated equilibrium which enhances performance of users. There are a few papers that find optimal solution for energy optimization using correlated equilibrium. An energy efficient resource allocation game is formulated in [11] while a cooperative policy selection game is formulated in [12] which uses correlated equilibrium to achieve energy efficiency in ad hoc networks. A reinforcement learning based method for power grids using correlated equilibrium is studied in [13].

Collision detection and resolution is a deeply researched area in robotics for both ground and aerial vehicles. The ground vehicles can adopt a wide variety of collision avoidance algorithms compared to the aerial vehicles because of the fact that they can carry a wide range of sensors and sufficient power source to aid safe traversal apart from having better control over their motion. The aerial robots, on the other hand, need to arrive at a trade-off between the payload it can carry and its endurance time. Since the unmanned systems use 'sense and avoid' methods for their safety, manned aircraft are in better position when it comes to collision avoidance as they employ 'see and avoid' strategies. In general, better algorithms are required for all class of vehicles for precise and efficient avoidance. A variety of algorithms exist addressing the problem which could be broadly classified into geometric methods, potential field based methods, optimization methods and others. Collision cone [14] and velocity obstacle [15] are algorithms belonging to the first while [16-18] gives avoidance using the second category of avoidance methods. Optimization based avoidance method is adopted in [19, 20]. There are other methods like forbidden velocity map [21], avoidance map [22, 23], bearing based method [24] which are derivatives of the methods in earlier classes. The avoidance problem has also been formulated using game theory. Multi-agent collision avoidance is modeled as a differential game and Nash equilibrium solutions are found in [25]. Nash equilibrium approach to study optimal control problems including collision avoidance is given 
in [26]. Apart from these, there is not much literature formulating the problem of UAV collision avoidance using game theory. In a conflict resolution system which is semi-autonomous, it is important to make sure that the vehicles involved are handled in a fair manner. Addressing the problem by the concept of correlated equilibrium ensures that every participating agent receives commands that optimizes its payoff. This paper proposes a novel game theoretic formulation for conflict resolution among UAVs using the concept of correlated equilibrium. In contrast to the other game theoretic methods, this paper introduces the idea of correlated equilibrium based conflict resolution system for UAVs which acts as a high level intelligence in avoidance decision making. This method is computationally less taxing and can be used for UAV conflict resolution irrespective of the collision avoidance algorithm employed.

The paper is organized as follows: Section [I gives an outline on the concept of correlated equilibrium and collision avoidance, Section III] details the underlying methodology of applying correlated equilibrium for conflict resolution. Section IV gives the simulation results and Section $\mathrm{V}$ concludes the paper.

\section{Conflict Resolution via Correlated Equilibrium}

\section{Correlated Equilibrium}

Consider an $n$ person game for which the strategy set is represented as $S=\left\{s_{1}, s_{2}, \ldots, s_{n}\right\}$, where, $s_{1}, s_{2}, \ldots, s_{n}$ represent the strategies of each of the players. Let the payoff corresponding to this game be represented as $P\left(s_{1}, s_{2}, \ldots, s_{n}\right)$. Let $\Delta$ be any function which lures the $i$ th player to change its strategy to $\Delta\left(s_{i}\right)$ when instructed to play $s_{i}$. The probability distribution $\Gamma(S)$ over the strategy set $S$ is in correlated equilibrium if for every $i \in n$,

$$
\int_{S} P_{i}(S) d \Gamma(S) \geq \int_{S} P_{i}\left(\Delta\left(s_{i}\right), S_{-i}\right) d \Gamma(S)
$$

where, $S_{-i}$ represents the strategy set for all others except for the $i$ th player and $P_{i}$ represents the payoff of $i^{t h}$ player. If all the players follow what is suggested, then the resultant $n$-tuple strategy $S$, is in correlated equilibrium. Eqn. (1) in turn means that none of the players gain by modifying their strategies which are in correlated equilibrium. While Nash equilibrium solution is NP-hard, correlated equilibrium only requires solving linear inequalities [27].

Consider Table 1. which shows the payoff matrix of a two player non-zero sum game. Player A chooses rows D or C while player B chooses columns D or C. The numbers in the payoff matrix $\left(a_{i}, a_{j}\right)$ represent the measure of gain or loss that each of the players incur while choosing a particular action pair. Both the players are utility maximizers. For the entries satisfying $0 \leq a_{1}<a_{2}<a_{3}<a_{4}$, the pure strategy Nash equilibrium are (D, C) and (C, D) while for mixed strategy Nash equilibrium, player A mixes his action with probability $(q, 1-q)$; where, $q$ is given by

$$
q=\frac{a_{4}-a_{3}}{a_{2}+a_{4}-a_{3}+a_{1}}
$$



Player B
Player B
\begin{tabular}{ccccc|} 
& & \multicolumn{1}{c}{$\mathrm{D}$} & \multicolumn{1}{c}{$\mathrm{C}$} \\
\cline { 3 - 4 } Player A & $\mathrm{D}$ & $a_{1}, a_{1}$ & $a_{4}, a_{2}$ \\
\cline { 3 - 4 } & $\mathrm{C}$ & $a_{2}, a_{4}$ & $a_{3}, a_{3}$ \\
\cline { 3 - 4 } & & &
\end{tabular}
D $\mathrm{C}$
Player A

\begin{tabular}{l|c|c|}
\multicolumn{1}{c}{} & \multicolumn{1}{c}{$\mathrm{D}$} & \multicolumn{1}{c}{$\mathrm{C}$} \\
\cline { 2 - 3 } $\mathrm{D}$ & $p_{1}$ & $p_{2}$ \\
\cline { 2 - 3 } $\mathrm{C}$ & $p_{3}$ & $p_{4}$ \\
\cline { 2 - 3 } & &
\end{tabular}

Table 1 An example
Table 2 Strategy profile for the example

Now, assume that there is some third party or a semi-automated system which chooses a strategy pair with some probability $p$. Each player receives the information about this strategy pair from this external entity and implements the strategy presented to it. Given this, there exists a conditional probability associated with other players' strategies. The strategy recommended by the third party would be optimal given this conditional probability. Table 2 gives a representative probability distribution for this two player game. For this distribution to be in correlated equilibrium, the following equations should hold:

$$
\begin{aligned}
a_{1} p_{1}+a_{2} p_{3} & \geq a_{4} p_{1}+a_{3} p_{3} \\
a_{4} p_{2}+a_{3} p_{4} & \geq a_{1} p_{2}+a_{2} p_{4} \\
a_{1} p_{1}+a_{2} p_{2} & \geq a_{4} p_{1}+a_{3} p_{2} \\
a_{4} p_{3}+a_{3} p_{4} & \geq a_{1} p_{3}+a_{2} p_{4} \\
p_{i} \geq 0 & \Sigma p_{i}=1, i=1, \ldots, 4
\end{aligned}
$$

The expected utility associated with each of the players are

$$
\begin{aligned}
& E P(A)=p_{1} a_{1}+p_{2} a_{4}+p_{3} a_{2}+p_{4} a_{3} \\
& E P(B)=p_{1} a_{1}+p_{2} a_{2}+p_{3} a_{4}+p_{4} a_{3}
\end{aligned}
$$

The expected payoff associated with the players in correlated equilibrium, (8)-99, would be at least as good as the Nash equilibrium payoff, as computed using (2). Introducing such correlated randomness into the game could result in better outcome for all the players. The suggestions of the external agent would be such that the players will not gain by deviating from the recommendation. Eqns. (3)-(7) are used to compute the candidate probabilities, given the payoff matrix. Those optimizing some objective of the vehicle can be chosen from this set of probabilities and the resultant strategies will lead to correlated equilibrium. Game of chicken is a popular game theory example in the literature, used to demonstrate the idea of correlated equilibrium. In this, the strategies are suggested to the players by a third party. The third party could either be a human mediator or any equivalent model which could intelligently correlate the 
strategies. In fact, the payoff matrix considered in the example is precisely that of game of chicken. The actions $\mathrm{D}$ and $\mathrm{C}$ correspond to Dare and Chicken, respectively. For the players to follow the third party decision, $p_{1}$ would be equal to zero which makes sure that the third party never recommends 'Dare' to both the players and thus the players have confidence on the external agent.

\section{Collision Avoidance}

As discussed in Sec. I. different methods could be employed for local path planning when a collision threat is detected by a vehicle. An efficient avoidance algorithm should be computationally light, have quick response with minimum information or sensing capability and preferably optimal in all possible aspects like avoidance time, power requirement, etc. The avoidance problem changes significantly according to the nature of response of the incoming threats. When there is information interchange between the vehicles, they could carry out avoidance benefiting each other. ADS-B could be a sensor to implement cooperative collision avoidance. There could be another case in which one or both of the vehicles tend to be non-cooperative, because the vehicles are either not communicating among themselves or one among the agents is communicating while the other is not responding. In such situations, the algorithm needs to guarantee successful avoidance. Incoming threats could also be detected by several onboard sensors like LIDAR, ultrasound sensor, camera, etc. But those obstacles which are within the range and field of view of these sensors could only be detected. Carrying long range and wide FOV sensors comes at the cost of higher power consumption and reduced flight time. In such scenario, detection relying on onboard sensors alone might not confirm safety.

The following section gives the details of CONCORD, the proposed UAV conflict resolution system which utilizes the concept of correlated equilibrium.

\section{CONCORD as a Generic Conflict Resolution System}

Consider a pair of UAV traveling along paths which are in collision course. Assume that their sensor and state information are available to a control station which is monitoring the states and various parameters of the vehicles. Given this information, the danger of collision could be predicted by the control station well ahead of time. Due to the limited sensing capabilities of unmanned systems, it is quite likely that the vehicles would know about the incoming threat much later than the control station. In this case, the intelligence support from the control station can effectively provide instructions for the vehicles to avoid colliding. The block schematic of the proposed CONCORD system is given in Fig. 1 The UAVs traverse in the regulated airspace and transmit periodically to the control station. CONCORD would be the subsystem for any typical UAV traffic management system which would usually consist of other subsystems like UAV health and sensor check, routing, take off/land permissions etc. It should be noted that this work is focused, not about the details of the avoidance algorithm as such but on the implementation of the support system, the performance of which leads to a correlated equilibrium. For a pair of UAVs in collision course, the following scenarios could be 


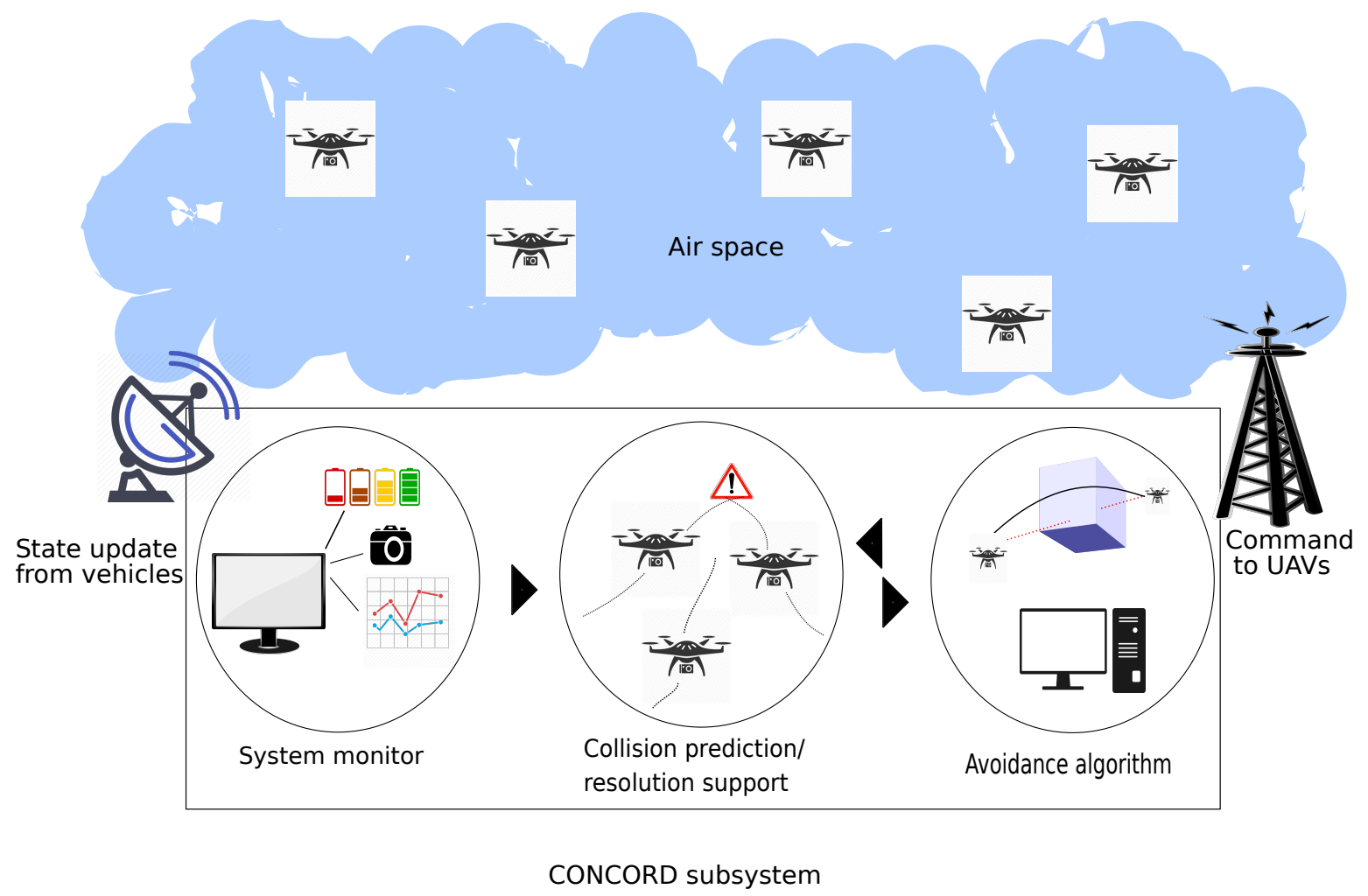

Fig. 1 Simplified block schematic of the proposed UAV CONCORD system

considered.

\section{Cooperative avoidance}

As discussed in Section [I], this is one class of avoidance scenario in which vehicles fully exchange information among each other and the avoidance strategies could be formulated in an optimal way. At the instant when the control station predicts collision between the UAVs, CONCORD is invoked. The avoidance module uses the current instant information of the vehicles like position, orientation, speed, etc., to compute various parameters like the avoidance maneuver and instant of the maneuver application. The vehicles could be considered as players and the avoidance problem, the game. The players could be minimizers in terms of fuel, time, etc. A payoff function could be constructed and also an equivalent payoff matrix for the game. This matrix would be dynamic in nature as it depends on the system states.

Now, assume that the intelligence which suggests the UAVs to perform certain avoidance maneuvers follows a particular probability distribution to do so. Since the vehicles are cooperative and the control station keeps track of the vehicle states and parameters, the suggested evasion strategy would be the best for the given pair of vehicles. This, however, does not preclude the UAVs from deviating from the control station command. In other words, if the control station utilizes a probability distribution to prescribe avoidance strategies for two UAVs in collision course and the 


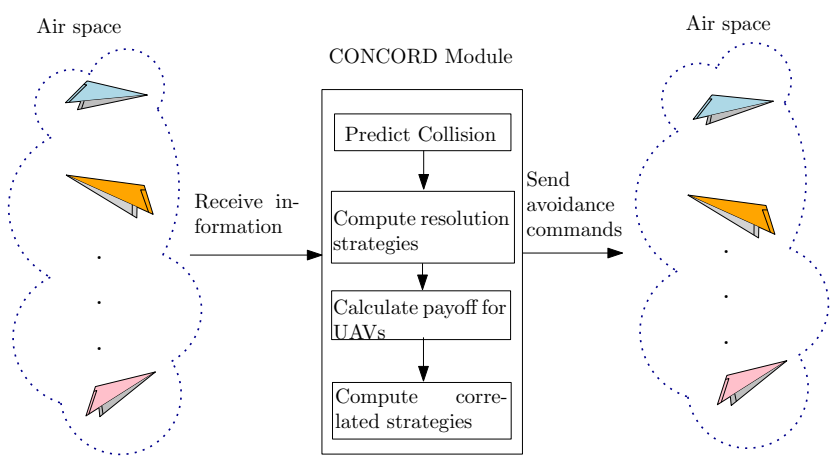

Fig. 2 Cooperative avoidance loop in CONCORD

vehicles do not deviate from the prescribed maneuvers, then the distribution associated with the avoidance game is in correlated equilibrium. For the pair of UAVs, this would mean that the expected value (e.g., fuel expenditure) associated with their game (avoidance process) in the long run would settle to an equilibrium in the correlated strategy (avoidance maneuver) and corresponding payoff (resultant expenditure) would be optimal for both the vehicles involved in the game. The schematic diagram representing the cooperative avoidance mechanism is as shown in Fig. 2

\section{Non-cooperative avoidance}

Another class of avoidance is that of non-cooperative UAVs. The vehicles might be tracked by the control station but the information interchange could be one way and not mutual. The inability to access or send information would make the avoidance problem more complex. The control station can neither suggest avoidance strategies nor receive the response of the vehicle. In such scenarios, with the assumption that the non-cooperative UAV takes no action to avoid collision, the best collision avoidance strategy for the cooperative UAV is computed. This would reach a correlated equilibrium if the cooperative vehicle obeys the strategy suggested by the control station and the non-cooperative one does not deviate from its course, every time such a scenario occurs. There could be cases where vehicles transmit information but remain unresponsive to the avoidance maneuvers suggested by the control station. The information available could be used to compute avoidance strategies by estimating the non-cooperating agent's motion. Correlated equilibrium could not be achieved if the non-cooperating UAV deviates from the suggested avoidance maneuver. In such cases, the CONCORD conflict resolution system at the control station would compute the best avoidance maneuver for the worst possible response of the non-cooperating UAV and the UAV in communication with the control station, will obey the command of the control station to get out of the conflict situation safely. The flow of process for CONCORD is summed up by Algorithm 1 .

\section{Handling multiple UAVs}

When the situation demands support for multiple vehicles simultaneously, the decisions need to be made intelligently. For this, the collision prediction system would compute the 'time to minimum separation $\left(t_{m s}\right)$ ' for all the pairs of 

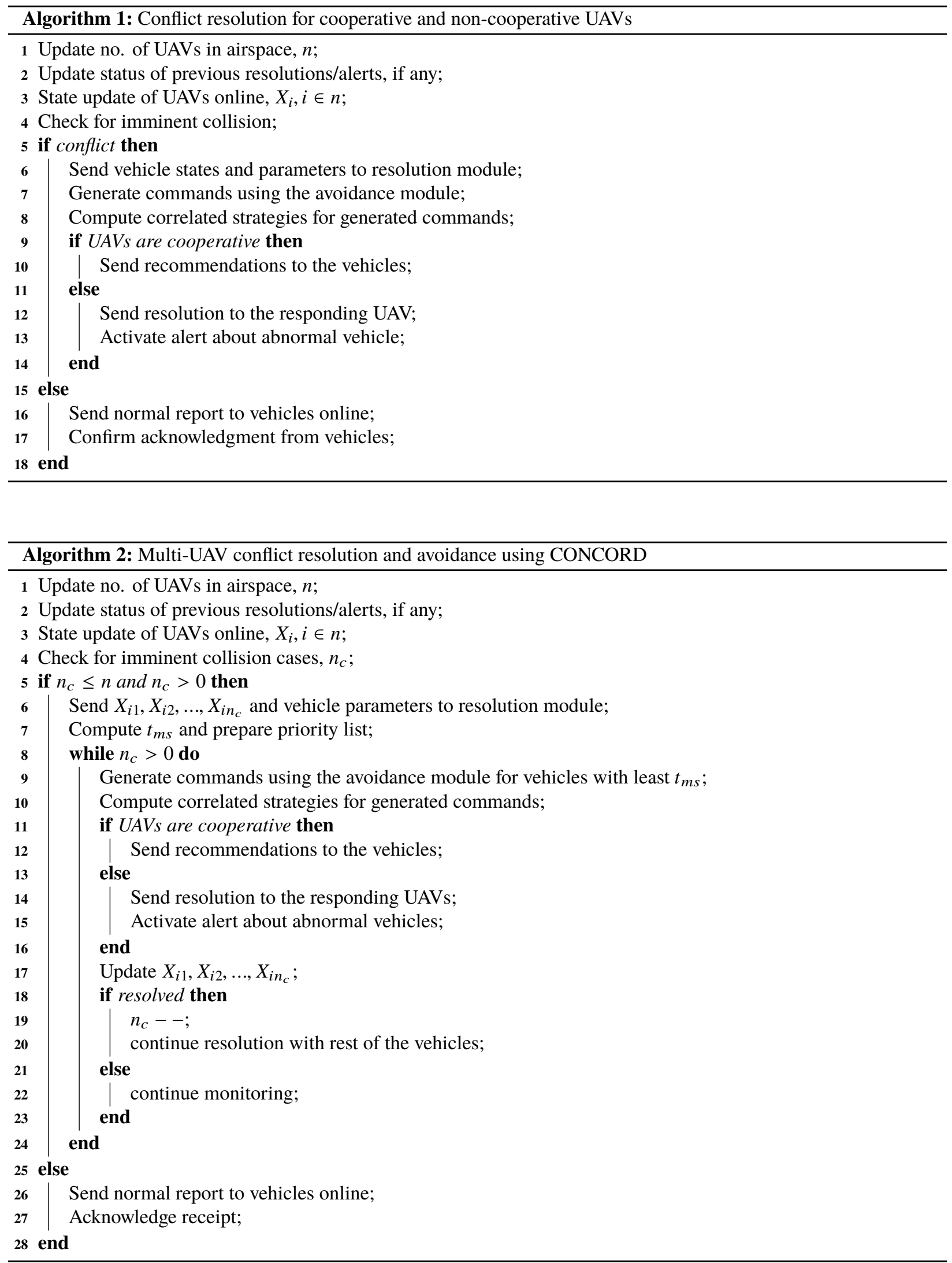
Vehicle 2

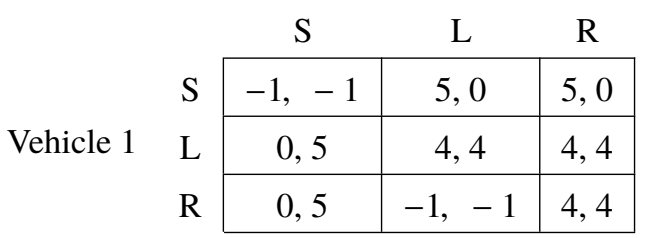

Table 3 Payoff matrix for two UAV conflict case
Vehicle 2

\begin{tabular}{cc|c|c|c|} 
& & \multicolumn{1}{c}{$\mathrm{S}$} & \multicolumn{1}{c}{$\mathrm{L}$} & \multicolumn{1}{c}{$\mathrm{R}$} \\
\cline { 3 - 5 } Vehicle 1 & $\mathrm{S}$ & $\mathrm{L}$ & 0.1174 & 0.1368 \\
\cline { 3 - 5 } & $\mathrm{N}$ & 0.2568 & 0.1174 & 0.0488 \\
\cline { 3 - 5 } & $\mathrm{R}$ & 0.2347 & 0 & 0.88 \\
\cline { 3 - 5 } & & & &
\end{tabular}

Table 4 Strategy distribution for two UAV conflict scenario

vehicles under consideration. Time to minimum separation is the time left from the current instant for any pair of UAVs to reach the shortest distance between them. This shortest distance would be predefined by the avoidance algorithm considering the shape, size and nature of the UAV. Based on $t_{m s}$, a priority list is prepared which is sent to the internal avoidance module to compute the collision resolution strategy set. The CONCORD layer would compute the correlated strategies and forward this to the vehicles. These would be recomputed according to the changing courses of the vehicles. The computed strategies would result in a correlated equilibrium once the vehicles follow the instructions from time to time, without fail. For multiple evasion maneuvers that the UAVs carry out, the expected payoff obtained for each of them would be optimal. There might be cases in which the computed avoidance maneuvers would lead to collision with other UAVs or the entire engagement scenario resulting in a deadlock. Such scenarios are rare and require further research, which is a part of future work in this area. The approach for multiple UAV conflict resolution could be summarized as in Algorithm 2 .

\section{Simulation Results}

In this section we consider illustrative examples for a pair of UAV and multiple UAVs, respectively, which are in collision course. For each of the cases, resolutions are computed by the available algorithm and the best ones are chosen using the proposed framework to bring the UAVs out of collision course. Consider the examples below.

Two UAV conflict scenario: Consider two aerial vehicles which are in collision course, as shown in Fig. 3 We assume that the avoidance scheme used generates three options, namely, left, straight and right. Although, in reality the UAVs will have more options such as graded left and right turns, and also options of going up or down, we consider only three options for the purpose of illustration. For each of these actions, the vehicles get some payoff which could be interpreted as their penalty/reward for collision or avoidance. This could be physically interpreted as the energy expenditure of the vehicles for avoidance or even the time spent for avoidance. This would be computed by the collision prediction system within CONCORD using the states of the vehicles. A representative payoff matrix is given in Table 3

The payoff values should be reasoned as follows: when both travel straight, both collide gaining negative payoff. 


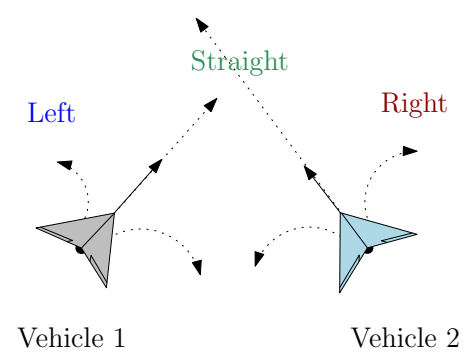

(a)

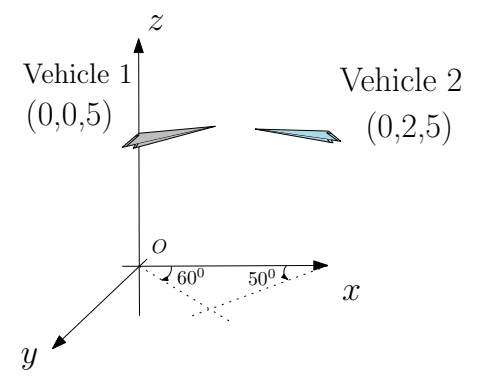

(b)

Fig. 3 (a) Vehicles in collision course with chosen action set (b) Pair of UAVs in 3D

When one goes straight and other takes a turn, collision is avoided but the one which went straight expended no effort which gives it higher payoff than the one which turned. When both turn, collision is avoided and both have equal payoff. All action pairs leading to collision are given negative payoff. In a realistic scenario, the actions left, straight and right would be replaced by those control inputs suggested by the avoidance module, which are within the actuation limits of the respective vehicles. For the set of actions considered and the associated payoff values in Table 3, the constraints for correlated strategies are formulated similar to (3)-(7). Now, solving these constraints result in the set of correlated probability distributions. One such distribution from among the set is given in Table 4 The values are chosen such that the probabilities corresponding to $(\mathrm{S}, \mathrm{S})$ and $(\mathrm{R}, \mathrm{L})$ are zero. This implies that the commands computed by the avoidance algorithm are confirmed safe and beneficial for both the players by the decision making outer layer when the correlated distributions are computed. Given the recommendation to play a particular action pair, the distribution is said to be in correlated equilibrium if no vehicle deviates from the given instruction. With the given distribution, the expected payoff of vehicle 1 in the above example is $E P(V 1)=5 \times 0.1174+4 \times 0.1174+5 \times 0.1368+4 \times 0.0488+4 \times 0.088=$ 3.4743 , which is more than the Nash equilibrium payoff. The expected payoff of vehicle 2 sums up to 2.2878 . Thus, the UAVs could be out of collision course by following the recommendations from the CONCORD system with improved benefit. Once the UAVs are out of collision course, an efficient re-planning could be done to redirect its path to the target location without ending up in collision course again. Fig. 4 (a) shows the convex hull of Nash equilibrium and correlated equilibrium payoffs. It can be seen that the set of correlated equilibrium strategies is the super set of Nash equilibrium strategies. The maximum payoff achieved by correlating is $(3,3)$ which provides a better utility for both the players.

Now, consider another case as in Fig. 3 b), which are in collision course. Let the avoidance scheme commands lateral acceleration as in [23] or [24]. For the given initial conditions at which collision is predicted, the computed avoidance command is found to be $u_{v 1}=[6.5,10] \mathrm{m} / \mathrm{s}^{2}$ and $u_{v 2}=[-6.5,-10] \mathrm{m} / \mathrm{s}^{2}$. Let us assume that the CONCORD scheme recommends the vehicles so as to optimize the energy spent. Table 5 gives the energy spent in avoidance (considering unit mass) for each control pair. The negative payoff represent those cases leading to collision. The control pairs are chosen such that possibility of one of the vehicles not applying any maneuver is also considered. The correlated 


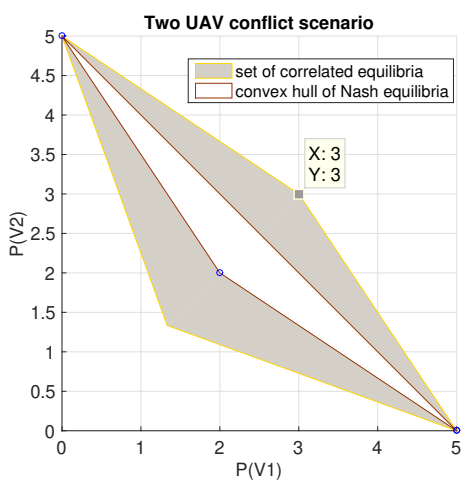

(a)

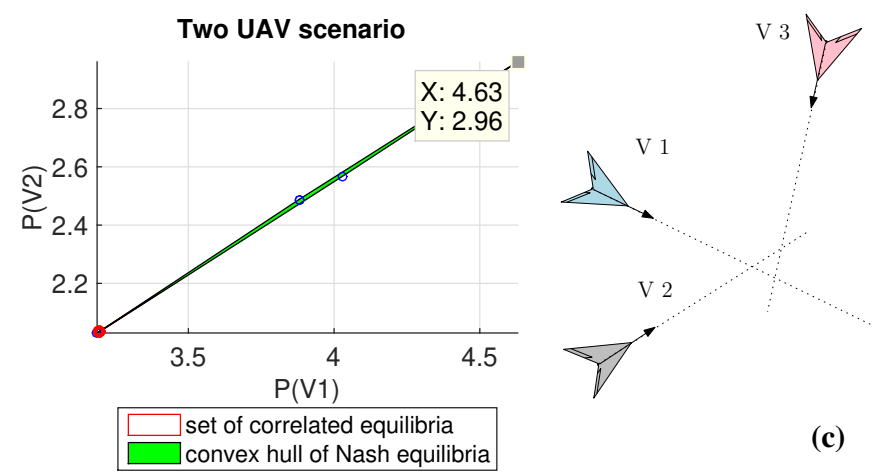

(b)

Fig. 4 (a) Convex hull of Nash equilibria and correlated equilibria for the two UAV conflict scenario (b) The two UAV scenario where correlated equilibrium gives same benefit as Nash equilibrium (c) Multi UAV scenario

Vehicle 2

\begin{tabular}{cc|c|c|c|} 
& \multicolumn{2}{c}{0} & \multicolumn{1}{c}{-5} & -10 \\
\cline { 3 - 5 } Vehicle 1 & 6.5 & $-10,-10$ & $-10,-10$ & $3.88,2.488$ \\
\cline { 3 - 5 } & 8 & $-10,-10$ & $4.63,2.96$ & $3.512,2.248$ \\
\cline { 3 - 5 } & 10 & $4.5,2.88$ & $4.0125,2.56$ & $3.138,2.008$ \\
\cline { 3 - 5 } & & & &
\end{tabular}

Vehicle 2

\begin{tabular}{|c|c|c|c|c|}
\hline Vehicle 1 & & 0 & -5 & -10 \\
\hline \multirow{3}{*}{ Vehicle 1} & 6.5 & 0 & 0 & 0.0239 \\
\hline & 8 & 0 & 0 & 0.0223 \\
\hline & 10 & 0.04 & 0.021 & 0.88 \\
\hline
\end{tabular}

Table 5 Payoff matrix for the energy optimization case
Table 6 Strategy distribution for the energy optimization case

strategies are computed and one among the distribution is as in Table 6. It is found that the expected payoff is same as Nash equilibrium payoff as shown in Fig. 4 b). This implies that there could be cases in which the expected payoff upon correlation could be same as the Nash equilibrium payoff. Nevertheless, the CONCORD layer mediates to make sure that the vehicles remain safe in an optimal way.

Multiple vehicle conflict scenario: In this case, multiple vehicles are in collision course. Consider the scenario as shown in Fig. 4(c). The conflict resolution system computes the $t_{m s}$ associated with each pair of UAVs, which are found to be in order $t_{m s}^{12}<t_{m s}^{13}<t_{m s}^{23}$, where, superscripts represent the vehicle pairs. The avoidance priorities are now assigned based on the $t_{m s}$ values. Since UAVs 1 and 2 have the least $t_{m s}$ or higher risk of collision, conflict resolution for vehicles 1 and 2 are made first. The scenario is similar to the two UAV case considered earlier and for the same action set that the vehicles exercised, further conflict resolution need not be done except for the action pairs $(\mathrm{S}, \mathrm{R}),(\mathrm{S}, \mathrm{L}),(\mathrm{L}, \mathrm{S})$ and $(\mathrm{R}, \mathrm{S})$. This is because, the action pairs except for $(\mathrm{S}, \mathrm{R}),(\mathrm{S}, \mathrm{L}),(\mathrm{L}, \mathrm{S})$ and $(\mathrm{R}, \mathrm{S})$ put the vehicles out of collision course for rest of the time. But, for the first two action pairs, vehicles 1 and 3 will remain in collision course while for the third and fourth choices, vehicles 2 and 3 continue in collision course. For either of the 


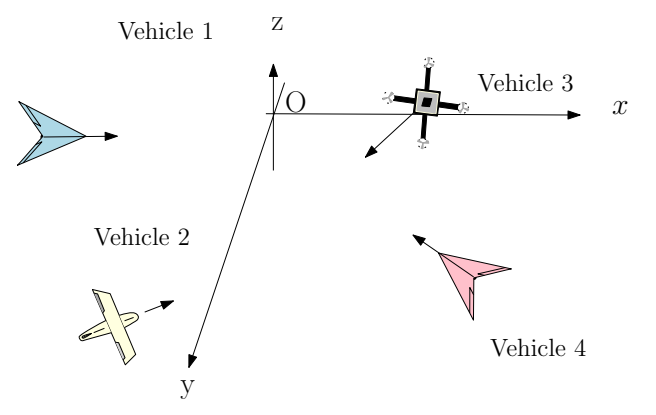

Fig. 5 Typical multi-UAV scenario

\begin{tabular}{c|c|c|c|} 
Action set & $a_{1}$ & $\ldots$ & $a_{i}$ \\
$a_{1}$ & $P_{1,1}^{v 1}, P_{1,1}^{v 2}, \ldots, P_{1,1}^{n_{c d}}$ & $\ldots$ & $P_{1, i}^{v 1}, P_{1, i}^{v 2}, \ldots, P_{1, i}^{n_{c d}}$ \\
\cline { 2 - 4 }$a_{2}$ & $P_{2,1}^{v 1}, P_{2,1}^{v 2}, \ldots, P_{2,1}^{n_{c d}}$ & $\ldots$ & $P_{2, i}^{v 1}, P_{2, i}^{v 2}, \ldots, P_{2, i}^{c_{c d}}$ \\
\cline { 2 - 4 } & $\vdots$ & $\ldots$ & $\vdots$ \\
\cline { 2 - 4 }$a_{j}$ & $P_{j, 1}^{v 1}, P_{j, 1}^{v 2}, \ldots, P_{j, 1}^{n_{c d}}$ & $\ldots$ & $P_{j, i}^{v 1}, P_{j, i}^{v 2}, \ldots, P_{j, i}^{n_{c d}}$ \\
\cline { 2 - 4 } & \multicolumn{2}{|c}{} &
\end{tabular}

Table 7 Payoff for multi UAV conflict scenario

scenarios, the avoidance algorithm would compute a pure solution of $(\mathrm{L}, \mathrm{R})$ or $(\mathrm{R}, \mathrm{L})$. This ensures that all the vehicles are out of the collision course. Here, the strategy correlation is applied for the first pair of vehicles alone as the third engagement between vehicles 2 and 3 has only one solution, for the action set considered. In actual scenarios, the decision making layer acts as a mediator which instructs the vehicles to take necessary action irrespective of the nature of their communication. This benefits the system in terms of safety, energy expenditure and makes it robust. In general, multi-agent avoidance resolution could be done independently or dependently. This means that, for certain scenarios resolving collision between a pair of agents would result in another conflict scenario. In such cases, the avoidance action should be taken by each vehicle until all are out of collision course. This aspect is dealt by the avoidance module and desired control actions are computed and passed on to the decision making layer. Now, the decision making layer receives the avoidance module input which consists of $n_{c}$ vehicles in collision course out of which $n_{c i}$ collisions could be independently resolved and $n_{c d}$ collisions needs dependent solving. The $n_{c i}$ cases could be solved similar to the discussion above where the payoff matrices are computed and in turn the correlated probabilities. Then, the CONCORD layer suggests actions pairs with certain probability distribution to be applied within next $\delta t_{a}$ s, for successful avoidance. The time $t+\delta t_{a}$ is the maximum time up to which the vehicles can maintain their current velocity without violating the safe separation between the vehicles. Thus, the vehicles execute the suggested action and attain correlated equilibrium. For the $n_{c d}$ vehicles, using the set of actions, the payoff matrix could be computed as one shown in Table 7 This is the abstract representation of payoff matrix for $n_{c d}$ vehicles. It is assumed that there are $i \times j$ action pairs available for the vehicles for avoidance. Now, the probabilities could be computed as earlier and resolutions could be made.

\section{Conclusions}

In this paper, a novel UAV conflict resolution system called CONCORD is proposed which aims at implementing a semi-automated ATC mandated system that would aid collision free traversal for vehicles in a given airspace. The concept of correlated equilibrium is used to design the conflict resolution system which allows the problem to be formulated using game theory. The proposed method is more realistic than the Nash equilibrium solution concept and 
optimizes the payoff for all the players while ensuring almost no risk and higher levels of safety. The proposed system would help the UAV system over come several of their shortcomings like endurance limits, communication constraints, heterogeneity in onboard sensor suit etc. Simulation results demonstrate how the decision layer could implement any avoidance algorithm and could direct the UAVs in optimal way. Applicability of the concept to multi-vehicle scenario is also briefed in this work. Further study on scalability of the proposed method is one among the future extensions of the same.

\section{Acknowledgments}

The first two authors (Lima Agnel Tony and Debasish Ghose) acknowledge partial funding support from the EPSRC-GCRF project (EP/P02839X/1).

\section{References}

[1] K. M. Fahey and M. J. Miller, “Unmanned systems integrated road-map 2017-2042,” Department of Defense, Technical Report, 2017.

[2] P. Kopardekar, "Enabling Autonomous Flight and Operations in the National Airspace," NASA Report ARC-E-DAA-TN72470, 2019.

[3] M. Erdelj et. al, "Help from the sky: Leveraging UAVs for disaster management," IEEE Pervasive Computing, vol.16, issue 1, pp. 24-32, 2017.

[4] J. F. Nash, "Equilibrium points in n-person games," In Proceedings of the National Academy of Sciences, vol. 36, issue 1, pp. 48-49, 1950.

[5] R. J. Aumann, "Subjectivity and correlation in randomized strategies," Journal of Mathematical Economics, vol. 1, pp. 67-96, 1974.

[6] R. J. Aumann, “Correlated equilibrium as an expression of Bayesian rationality,” Econometrica: Journal of the Econometric Society, pp. 1-18, 1987.

[7] J. W. Huang and V. Krishnamurthy, "Cognitive base stations in LTE/3GPP femtocells: A correlated equilibrium game-theoretic approach,” IEEE Transactions on Communications, vol. 59, issue 12, pp. 3485-3493, 2011.

[8] Z. Han, C. Pandana, and K. R. Liu, "Distributive opportunistic spectrum access for cognitive radio using correlated equilibrium and no-regret learning," In Proceedings of Wireless Communications and Networking Conference, pp. 11-15, 2007.

[9] J. Zheng, Y. Cai and D. Wu, "Subcarrier allocation based on correlated equilibrium in multi-cell OFDMA systems," EURASIP Journal on Wireless Communications and Networking, vol. 1, p. 233, 2012.

[10] B. Wang, Z. Han, and K. R. Liu, "Peer-to-peer file sharing game using correlated equilibrium," In 43rd Annual Conference of Information Sciences and Systems, CISS 2009, pp. 729-734, 2009. 
[11] D. Wu, L. Zhou and Y. Cai, "Energy-efficient resource allocation for uplink orthogonal frequency division multiple access systems using correlated equilibrium," IET Communications, vol. 6, pp. 659-667, 2012.

[12] D. Wu, J. Zheng, Y. Cai, L. Yang and W. Yang, "Cooperation policy selection for energy-constrained ad hoc networks using correlated equilibrium,” In International Conference on Green Communications and Networking, pp. 161-170, 2011.

[13] T. Yu, H. Z. Wang, B. Zhou, K. W. Chan and J. Tang, "Multi-agent correlated equilibrium Q( $\lambda$ ) learning for coordinated smart generation control of interconnected power grids," IEEE Transactions on Power Systems, vol. 30, issue 4, pp. 1669-1679, 2015.

[14] A. Chakravarthy and D. Ghose, "Obstacle avoidance in a dynamic environment: A collision cone approach,” IEEE Transactions on Systems, Man and Cybernetics- A., Systems and Humans, vol. 28, issue 5, pp. 562-574, 1998.

[15] P. Fiorini and Z. Shiller , "Motion planning in dynamic environments using velocity obstacles," The International Journal of Robotics Research, vol. 17, issue 7, pp. 760-772, 1998.

[16] Z. Wu, G. Hu, L. Feng, J. Wu and S. Liu, "Collision avoidance for mobile robots based on artificial potential field and obstacle envelope modelling," Assembly Automation, vol. 36, issue 3, pp. 318-332, 2016.

[17] D. Gonon, D. Jud, P. Fankhauser and M. Hutter, "Safe Self-collision Avoidance for Versatile Robots Based on Bounded Potentials," In Field and Service Robotics, pp. 19-33, 2018.

[18] G. Fedele, L. D’Alfonso, F. Chiaravalloti and G. D’Aquila, “Obstacles Avoidance Based on Switching Potential Functions,” Journal of Intelligent and Robotic Systems, vol. 1, pp. 1-19, 2018.

[19] B. Penin, P. R. Giordano and F. Chaumette, "Vision-based reactive planning for aggressive target tracking while avoiding collisions and occlusions," IEEE Robotics and Automation Letters, vol. 3, issue 4, pp. 3725-3732, 2018.

[20] D. Fox, ,W. Burgard and S. Thrun, “The dynamic window approach to collision avoidance," IEEE Robotics and Automation Magazine, vol. 4, issue 1, pp. 23-33, 1997.

[21] B. Damas, and J. Santos-Victor, “Avoiding moving obstacles: the forbidden velocity map," In International Conference on Intelligent Robots and Systems, IROS, pp. 4393-4398, 2009.

[22] L. A. Tony, D. Ghose and A. Chakravarthy, "Avoidance maps: A new concept in UAV collision avoidance," In International Conference on Unmanned Aerial Systems, pp. 1483-1492, 2017.

[23] L. A. Tony, D. Ghose and A. Chakravarthy, "Precision UAV collision Avoidance for computationally efficient avoidance maps," In AIAA Guidance, Navigation, and Control Conference, p. 0875, 2018.

[24] L. Schmitt and W. Fichter, "Collision-avoidance framework for small fixed-wing unmanned aerial vehicles," Journal of Guidance, Control, and Dynamics, vol. 37, no. 4, pp. 1323-1329, 2014.

[25] T. Mylvaganam, S. Mario and A. Alessandro, "A differential game approach to multi-agent collision avoidance," IEEE Transactions on Automatic Control, vol. 62, issue 8, pp. 4229-4235, 2017. 
[26] A. Dreves and G. Matthias, "A generalized Nash equilibrium approach for optimal control problems of autonomous cars," Optimal Control Applications and Methods, vol. 39, issue 1, pp. 326-342, 2018.

[27] I. Gilboa and E. Zemel, "Nash and correlated equilibria: Some complexity considerations," Games and Economic Behavior, vol. 1, issue 1, pp. 80-93, 1989. 